\title{
An easy procedure to transform the ratio of two polynomials of first degree into Michaelis-Menten-type equations. Application to the ordered Uni Bi enzyme mechanism ${ }^{\star}$
}

\author{
Rui Fontes ${ }^{\star}$, João Meireles Ribeiro and Antonio Sillero ${ }^{\varpi}$ \\ Instituto de Investigaciones Biomédicas Alberto Sols, UAM-CSIC, Departamento de Bioquímica, \\ Facultad de Medicina, Arzobispo Morcillo 4, E-28029 Madrid, Spain
}

Received: 3 November, 1999

Key words: enzyme equations, enzyme inhibition, enzyme kinetics, graphical presentations, rectangular hyperbolas

\begin{abstract}
It is not always clear that some equations affected by complicated factors can, actually, be interpreted as a ratio of two polynomials of first degree and so that they can be, in general, represented by rectangular hyperbolas. In this paper we present an easy procedure to rearrange those equations into Michaelis-Menten-type equations and so to make the aspects of these rectangular hyperbolas more clear, particularly for researchers familiar with general biochemistry. As an example, the method is applied to transform the classical rate equation of the Cleland's Ordered Uni Bi enzyme mechanism.
\end{abstract}

There are many biological phenomena that follow saturation kinetics, e.g. enzyme-catalyzed reactions, mediated transport through membranes, stimulation of cells by the binding of compounds to receptors, etc. Some equations related to these processes can be in-

\footnotetext{
Supported by grants from Dirección General de Investigación Científica y Técnica (PM95/0013 and PM98/0129, A.S.; PM97/0022, J.M.R.) and from Comunidad de Madrid (08.9/0004/98, to A.S.). R.F. was supported by a fellowship from Junta Nacional de Investigação Científica e Tecnológica (Portugal). During part of this work, J.M.R. held a postdoctoral fellowship from Consejería de Educación y Juventud, Junta de Extremadura (Spain; PRI9606B064), cofinanced by the Fondo Social Europeo.

${ }^{\not}$ Present address: Serviço de Química Fisiológica, Faculdade de Medicina, Universidade do Porto, Porto, Portugal.

Present address: Unidad de Bioquímica y Biología Molecular, Facultad de Medicina, Universidad de Extremadura, E-06080 Badajoz, Spain; e-mail: jribeiro@unex.es

${ }^{\otimes}$ Corresponding author: Dr. Antonio Sillero, Departamento de Bioquímica, Facultad de Medicina UAM, Arzobispo Morcillo 4, 28029 Madrid, Spain; phone: (34 91) 397 5413; fax: (34 91) 397 5353;

e-mail: antonio.sillero@uam.es
} 
terpreted as functions that correspond to hyperbolic graphs.

The Michaelis-Menten equation is a good example of a frequently used hyperbolic function $(v=f([S]))$ :

$v=\frac{V_{\max }[\mathrm{S}]}{K_{m}+[\mathrm{S}]}$

in which $v$ is the initial velocity in an enzyme-catalyzed reaction; $V_{\max }$ is the maximal velocity, i.e. the velocity attained at very high concentration of substrate [S]; $K_{\mathrm{m}}$ is the Michaelis constant and corresponds to the concentration of substrate at which $v=V_{\max } / 2$.

This equation follows a form that is called here equation of type 1 :

$\mathrm{y}=\frac{a \mathrm{x}}{b+\mathrm{x}}$

(type 1)

The rectangular hyperbola (y versus $\mathrm{x}$ ) described by an equation of type 1, crosses the origin of the axes and the equations for the horizontal and the vertical asymptotes are $\mathrm{y}=a$ and $\mathrm{x}=-b$, respectively.

From the mathematical point of view it is known that in functions of the form

$\mathrm{y}=\frac{n_{0}+n_{1} \mathrm{x}}{d_{0}+d_{1} \mathrm{x}}$

(type 2)

where $n_{0}, n_{1}, d_{0}$ and $d_{1}$ are constants, the plot $\mathrm{y}$ versus $\mathrm{x}$ is also a rectangular hyperbola provided that $d_{1} \neq 0$ and $n_{0} d_{1} \neq n_{1} d_{0}$. However, when the constants are complicated factors, it is not always clear that equations of type 2 actually represent hyperbolic functions.

In the course of an investigation on the effect of a modifier on the rate of an enzyme-catalyzed reaction (ref. [1]; accompanying paper), we handled complex equations of type 2 and arrived at a general approach making possible to transform those equations into another type (type 3; see below) that, because $\mathrm{y}=\frac{n_{0}}{d_{0}}+\frac{n_{1} d_{0} \mathrm{x}-n_{0} d_{1} \mathrm{x}}{d_{0}^{2}+d_{0} d_{1} \mathrm{x}}$

they resemble the classical Michaelis-Menten equation, more clearly show that they represent rectangular hyperbolas.

\section{EQUATION OF TYPE 3}

Equations of type 2 can be interpreted as a ratio of two polynomials of first degree and they can be converted into MichaelisMenten-type equations (type 3 ) by adding and subtracting $n_{0} / d_{0}$ :

$\mathrm{y}=\frac{n_{0}}{d_{0}}+\frac{n_{0}+n_{1} \mathrm{x}}{d_{0}+d_{1} \mathrm{x}}-\frac{n_{0}}{d_{0}}$

$y=\frac{n_{0}}{d_{0}}+\frac{\frac{n_{1} d_{0}-n_{0} d_{1}}{d_{0} d_{1}} \mathrm{x}}{\frac{d_{0}}{d_{1}}+\mathrm{x}}$

or, in general,

$\mathrm{y}=h+\frac{a \mathrm{x}}{b+\mathrm{x}}$

(type 3)

By comparing eqns. (4) and type 3 , the parameters $(h, a$, and $b)$ of the type 3 equation can be deduced:

$h=\frac{n_{0}}{d_{0}}, \quad a=\frac{n_{1} d_{0}-n_{0} d_{1}}{d_{0} d_{1}}=\frac{n_{1}}{d_{1}}-\frac{n_{0}}{d_{0}}$,

and $b=\frac{d_{0}}{d_{1}}$.

Equation of type 3 describes a rectangular hyperbola where $h$ is the value of the ordinate 
of the point where the hyperbola intercepts the vertical axis. The equation for the horizontal asymptote is $\mathrm{y}=a+h$ (i.e. $\mathrm{y}=n_{1} / d_{1}$ ) and that for the vertical asymptote is $\mathrm{x}=-b$. The value of $b$ can also be described as the value of $\mathrm{x}$ at which $\mathrm{y}=h+a / 2$. The value of $a$ is the difference between two values: the ordinate of the point where the horizontal asymptote intercepts the vertical axis and $h\left(\alpha=n_{1} / d_{1}-h\right)$.

Assuming $b$ as a positive value, as it occurs in the biological phenomena referred to above, one of the advantages of an equation of type 3 is that the sign of the parameter $a$ indicates when the hyperbola is ascendant $(a>0)$ or when it is descendant $(a<0)$; another advantage is that an equation of type 3 is more easily turned into a linear form (see below).

The procedure shown in eqns. (2-4) is valid when $d_{0} \neq 0$; i.e., when the vertical asymptote of the hyperbola is not the vertical axis itself. However, even in the case $d_{0}=0$, the equation for the vertical asymptote of the hyperbola can be obtained by evaluating the mathematical limit in an equation of type 3 : when $\mathrm{d}_{0}$ tends to zero, $b$ tends to zero.

In the particular case in which $n_{1}=0$ in an equation of type 2 , the horizontal asymptote of the rectangular hyperbola is the horizontal axis itself and the procedure shown in eqns. (2-4) is valid; in this case, in the corresponding equation of type 3 , the parameters $h$ and $a$ have the same absolute value but opposite signs.

As stated in the introduction, the equation of type 2 represents a hyperbola when $\mathrm{d}_{1} \neq 0$ and $n_{0} d_{1} \neq n_{1} d_{0}$. Actually, if the previous conditions do not apply, the equation corresponds to a straight line. In the case $d_{1}=0$, the value of the ordinate at the origin is $n_{0} / d_{0}$ and the value of the slope is $n_{1} / d_{0}$. In the case $n_{0} d_{1}=n_{1} d_{0}$, it is not so obvious that equation of type 2 represents a straight line. However, if its transformation into an equation of type 3 is attempted, following the procedure described above, it results in $a=0$, and so this straight line, that is parallel to the horizontal axis, may be viewed as a limit case between an ascendant rectangular hyperbola $(a>0)$ and a descendant one $(a<0)$.

In the accompanying paper [1], the mathematical procedure described in eqns. (2-4) has been systematically used to rearrange equations of type 2 into equations of type 3 ; examples are the rearrangements of eqns. (5), (10), (11), and (15a) into eqns. (5a), (10a), (11a), and (15), respectively (all of them numbered as in ref. [1]).

As an example, eqn. (5) is here rearranged into eqn. (6) (numbered 5 and 5a, respectively, in ref. [1])

$K_{e}=\frac{K_{x 1} K_{s 2}+K_{x 2}[\mathrm{~S}]}{K_{s 2}+[\mathrm{S}]}$

$K_{e}=K_{x 1}+\frac{\left(K_{s 2}+K_{x 1}\right)[\mathrm{S}]}{K_{s 2}+[\mathrm{S}]}$

The above procedure, as such or with minor variants, may be of general application. As an example, it is here applied to classical enzyme reaction rate equations deduced by Cleland [2].

\section{CLELAND EQUATIONS; THE ORDERED UNI BI CASE}

The following are examples of the formulas deduced by Cleland for the steady state assumption kinetics [2]:

Ordered Uni Bi mechanism:

$$
v=\frac{\left(k_{1} k_{3} k_{5} A-k_{2} k_{4} k_{6} P Q\right) E t}{\left(k_{2}+k_{3}\right) k_{5}+k_{1}\left(k_{3}+k_{5}\right) A+k_{2} k_{4} P+\left(k_{2}+k_{3}\right) k_{6} Q+k_{1} k_{4} A P+k_{4} k_{6} P Q}
$$


Ordered Bi Bi mechanism:

$$
\begin{aligned}
v= & \frac{\left(k_{1} k_{3} k_{5} k_{7} A B-k_{2} k_{4} k_{6} k_{8} P Q\right) E t}{k_{2}\left(k_{4}+k_{5}\right) k_{7}+k_{1}\left(k_{4}+k_{5}\right) k_{7} A+k_{3} k_{5} k_{7} B+k_{1} k_{3}\left(k_{5}+k_{7}\right) A B+k_{1} k_{4} k_{6} A P+k_{3} k_{5} k_{8} B Q+} \\
& +k_{2}\left(k_{4}+k_{5}\right) k_{8} Q+k_{2} k_{4} k_{6} P+\left(k_{2}+k_{4}\right) k_{6} k_{8} P Q+k_{1} k_{3} k_{6} A B P+k_{3} k_{6} k_{8} B P Q
\end{aligned}
$$

Ping Pong Bi Bi mechanism:

$$
\begin{aligned}
v= & \frac{\left(k_{1} k_{3} k_{5} k_{7} A B-k_{2} k_{4} k_{6} k_{8} P Q\right) E t}{k_{1}\left(k_{4}+k_{5}\right) k_{7} A+k_{3} k_{5} k_{7} B+k_{1} k_{3}\left(k_{5}+k_{7}\right) A B+k_{1} k_{4} k_{6} A P+k_{3} k_{5} k_{8} B Q+} \\
& +k_{2}\left(k_{4}+k_{5}\right) k_{8} Q+k_{2} k_{4} k_{6} P+\left(k_{2}+k_{4}\right) k_{6} k_{8} P Q
\end{aligned}
$$

where $k_{\mathrm{n}}$ are rate constants (whose significance is irrelevant in the context of this paper), $A$ and $B$ are concentrations of substrates, $P$ and $Q$ are concentrations of products and $E t$ is the concentration of enzyme; $v$ is the reaction rate for the conversion of $\mathrm{A}$ $(+\mathrm{B})$ into $\mathrm{P}+\mathrm{Q}$. When the reaction proceeds in the opposite direction $v$ has a negative value; in this paper $\mathrm{P}$ and $\mathrm{Q}$ will be called products independently of the macroscopic direction of the reaction.
The equations deduced by Cleland [2] can be viewed from a new perspective if the mathematical procedure described above is applied, as exemplified here for the case of the Ordered Uni Bi mechanism.

Equations (7-9) can be arranged to show more clearly that they fit the type 2 model, if the concentration of one of the substrates or one of the products replaces x. As examples, the eqns. $(10-12)$ were obtained by rearranging eqn. (7):

(i) variable $A(A=\mathrm{x}) ; P$ and $Q$ are assumed constants

$v=\frac{\left(-k_{2} k_{4} k_{6} P Q E t\right)+\left(k_{1} k_{3} k_{5} E t\right) A}{\left(\left(k_{2}+k_{3}\right) k_{5}+k_{2} k_{4} P+\left(k_{2}+k_{3}\right) k_{6} Q+k_{4} k_{6} P Q\right)+\left(k_{1}\left(k_{3}+k_{5}\right)+k_{1} k_{4} P\right) A}$

(ii) variable $P(P=\mathrm{x}) ; A$ and $Q$ are assumed constants

$v=\frac{\left(k_{1} k_{3} k_{5} A E t\right)+\left(-k_{2} k_{4} k_{6} Q E t\right) P}{\left(\left(k_{2}+k_{3}\right) k_{5}+k_{1}\left(k_{3}+k_{5}\right) A+\left(k_{2}+k_{3}\right) k_{6} Q\right)+\left(k_{2} k_{4}+k_{1} k_{4} A+k_{4} k_{6} Q\right) P}$ 
(iii) variable $Q(Q=\mathrm{x}) ; A$ and $P$ are assumed constants

$v=\frac{\left(k_{1} k_{3} k_{5} A E t\right)+\left(-k_{2} k_{4} k_{6} P E t\right) Q}{\left(\left(k_{2}+k_{3}\right) k_{5}+k_{1}\left(k_{3}+k_{5}\right) A+k_{2} k_{4} P+k_{1} k_{4} A P\right)+\left(\left(k_{2}+k_{3}\right) k_{6}+k_{4} k_{6} P\right) Q}$

Equations (10-12), arranged as type 2 equations, can now be transformed into equations of type 3 by applying the procedure deduced above. The method could also be used to analyze Ordered Bi Bi (eqn. 8) or Ping Pong Bi Bi (eqn. 9) mechanisms. However, only the Ordered Uni Bi mechanism will be analyzed here (eqn. 10).

Equation (13) is deduced from eqn. (10): $\quad v=h+\frac{a A}{b+A}$

where

$h=\frac{-k_{2} k_{4} k_{6} P Q E t}{\left(k_{2}+k_{3}\right) k_{5}+k_{2} k_{4} P+\left(k_{2}+k_{3}\right) k_{6} Q+k_{4} k_{6} P Q}$

$a=\frac{n_{1}}{d_{1}}-h=\frac{k_{1} k_{3} k_{5} E t}{k_{1}\left(k_{3}+k_{5}\right)+k_{1} k_{4} P}+\frac{k_{2} k_{4} k_{6} P Q E t}{\left(k_{2}+k_{3}\right) k_{5}+k_{2} k_{4} P+\left(k_{2}+k_{3}\right) k_{6} Q+k_{4} k_{6} P Q}$

$b=\frac{\left(k_{2}+k_{3}\right) k_{5}+k_{2} k_{4} P+\left(k_{2}+k_{3}\right) k_{6} Q+k_{4} k_{6} P Q}{k_{1}\left(k_{3}+k_{5}\right)+k_{1} k_{4} P}$

By analyzing eqns. (13-16) (see below), it is easy to visualize the aspect of the plot $v$ versus $A$, and the way it depends on $P$ and $Q$.

(1) $\mathrm{P}$ and $\mathrm{Q}$ are assumed constants; general aspects. Equation (13) shows that the plot $v$ versus $A$ is, in general, a rectangular hyperbola (Fig. 1; plot 1):

(a) The hyperbola intercepts the vertical axis below the origin of the axes $(h<0)$ when neither $P$ nor $Q$ are zero. When $P$ or $Q$ are zero, $h=0$ and the hyperbola crosses the origin of the axes (Fig. 1; plot 2). (b) The hyperbola is ascendant $(a>0$ and $b>0)$.

(c) The equation for the horizontal asymptote ( $\mathrm{y}=n_{1} / d_{1}$; see eqn. 17$)$ shows that, in general, the hyperbola tends to a positive value when $A$ tends to saturation (i.e. $A \rightarrow \infty$ ) and so the hyperbola crosses the horizontal axis.

$$
\frac{n_{1}}{d_{1}}=\frac{k_{3} k_{5} E t}{k_{3}+k_{5}+k_{4} P}
$$


The point where the hyperbola crosses the horizontal axis $(v=0)$ corresponds to chemical equilibrium; the value of $A$ at which chemical equilibrium is obtained $\left(\mathrm{A}_{\mathrm{eq}}\right)$ depends, obviously, on the values of $P$ and $Q$. The points of the hyperbola when $v$ is positive correspond to concentrations of $\mathrm{A}$ at which the reaction proceeds from $\mathrm{A}$ into $\mathrm{P}+\mathrm{Q}$, and the points when $v$ is negative, to concentrations of $\mathrm{A}$ at which the reaction proceeds from $\mathrm{P}+\mathrm{Q}$ into $\mathrm{A}$.

As all the three parameters $h, a$, and $b$ (eqn. 13) depend on both $P$ and $Q$, the actual aspect of the plot $v$ versus $A$ depends on the values of $P$ and $Q$. The effect produced by $P$ or $Q$ on this plot can be deduced by analyzing how the parameters $h, a$, and $b$ depend on their values (eqns. 14-16). Interestingly, these equations and the equation for the horizontal asymptote (17) recall equations of type 2 ; when they are, actually, equations of type 2 , a reasoning similar to that followed above can be made and the aspect of the corresponding plots visualized intuitively.

(2) Influence of $\mathrm{P}$ on the hyperbola parameters. If $P$ is considered the variable in eqns. (14), (16) and (17), it is easy to recognize them as equations of type 2; obviously, $n_{0}=0$ in eqn (14) and $n_{1}=0$ in eqn. (17). Equation (15) may be interpreted as a sum of two "hyperbolic equations" $\left[n_{1} / d_{1}+(-h)\right]$; a versus $P$ is not, in general $^{\#}$, a hyperbola (see Fig. 2).

Equations (14-17) can be rearranged using the procedure presented above:

$h=\frac{-\left(\frac{k_{2} k_{6} Q E t}{k_{2}+k_{6} Q}\right) P}{\left(\frac{\left(k_{2}+k_{3}\right) k_{5}+\left(k_{2}+k_{3}\right) k_{6} Q}{k_{2} k_{4}+k_{4} k_{6} Q}\right)+P}$

$a=\frac{n_{1}}{d_{1}}-h=\frac{\frac{k_{3} k_{5} E t}{k_{4}}}{\frac{k_{3}+k_{5}}{k_{4}}+P}+\frac{\left(\frac{k_{2} k_{6} Q E t}{k_{2}+k_{6} Q}\right) P}{\left(\frac{\left(k_{2}+k_{3}\right) k_{5}+\left(k_{2}+k_{3}\right) k_{6} Q}{k_{2} k_{4}+k_{4} k_{6} Q}\right)+P}$

$b=\frac{\left(k_{2}+k_{3}\right) k_{5}+\left(k_{2}+k_{3}\right) k_{6} Q}{k_{1}\left(k_{3}+k_{5}\right)}+\frac{\left(\frac{\left(k_{2}-k_{5}\right) k_{3}-\left(k_{2}-k_{5}\right) k_{6} Q}{k_{1}\left(k_{3}+k_{5}\right)}\right) P}{\frac{k_{3}+k_{5}}{k_{4}}+P}$

\footnotetext{
\#The addition of two equations that represent rectangular hyperbolas represents a rectangular hyperbola in the particular case when the vertical asymptotes of the two hyperbolas coincide.
} 


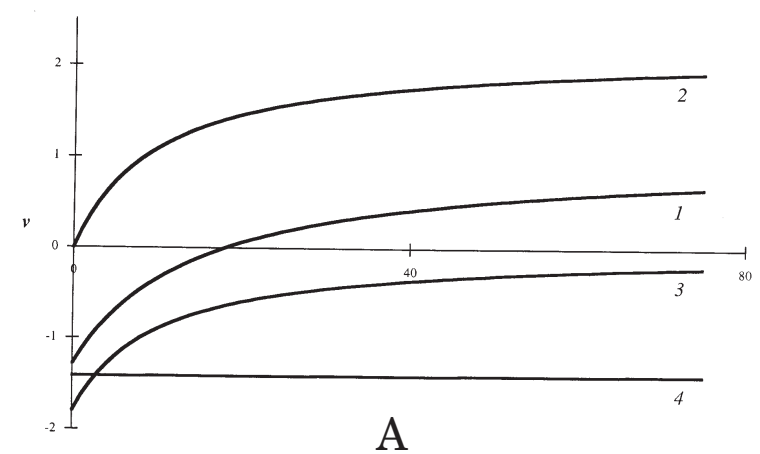

Figure 1. Influence of concentration of the substrate A on the reaction rate (initial velocity) in the Uni Bi enzyme mechanism.

Plot 1 represents the general case in which $P$ and $Q$ are nonzero and finite quantities; plot 2 displays the situation when $P$ or $Q$ are zero; in plot 3, $P$ is saturating and $Q \neq 0$; in plot $4, Q$ is saturating and $P \neq 0$. The graph has been drawn using the program Microsoft Excel 4.0 for Macintosh, assuming the following set of values for the rate constants: $k_{1}=2^{*} 10^{3}, k_{2}=2^{*} 10^{3}, k_{3}=3^{*} 10^{3}, k_{4}$ $=4^{*} 10^{3}, k_{5}=8^{*} 10^{3}$, and $k_{6}=6^{*} 10^{3}$; the dimensions of $k_{1}, k_{4}$ and $k_{6}$ are (concentration ${ }^{-1}$ time $^{-1}$ ) and those for $k_{2}, k_{3}$ and $k_{5}$ are (time ${ }^{-1}$ ); Et is always equal to $10^{-3}$. $P=3$ and $Q=6$ in plot $1 ; P=0$ and $Q=6$ in plot $2 ; P=1000$ and $Q=3$ in plot 3 ; and $P=3$ and $Q=5000$ in plot 4. A, $P, Q$ and $E t$ have the dimension of concentration and the enzyme reaction rate $(v)$ dimensions of (concentration time ${ }^{-1}$ ). Rate constants, $A, P, Q$ and $E t$ are defined as in ref. [2]. In all cases units are irrelevant and arbitrary.

$$
\frac{n_{1}}{d_{1}}=\frac{k_{3} k_{5} E t}{k_{3}+k_{5}}+\frac{-\frac{k_{3} k_{5} E t}{k_{3}+k_{5}} P}{\frac{k_{3}+k_{5}}{k_{4}}+P}
$$

If $Q$ is assumed constant, and a nonzero and finite quantity, from these equations it can be intuitively recognized that the plots $h$ versus $P, b$ versus $P$ and $n_{1} / d_{1}$ versus $P$ are rectangular hyperbolas (see Fig. 2). As $P$ increases from zero to saturation (i.e. $P \rightarrow \infty$ ) the value of $h$ decreases from zero to a finite negative quantity, the value of $b$ varies between two finite positive quantities, and the value of $n_{1} / d_{1}$ decreases from a finite positive quantity to zero.
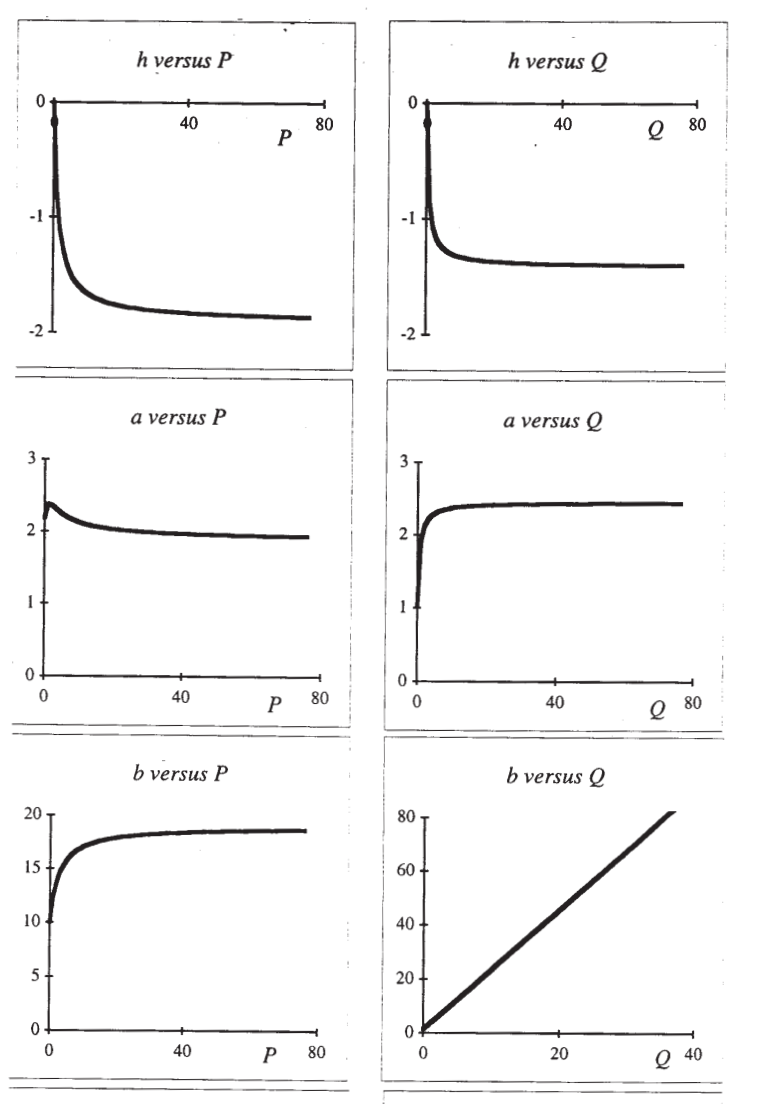

nl/dl versus $P$

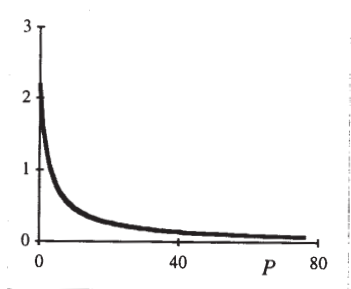

nl/dl versus $Q$

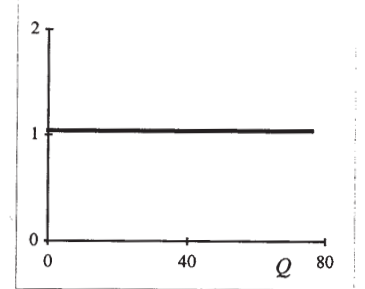

Figure 2. Influence of the concentration of $\mathbf{P}$ or $\mathbf{Q}$ on the reaction rate parameters in the Uni Bi enzyme mechanism.

At the left, plots $h$ versus $P$, a versus $P, b$ versus $P$, and $n_{1} / d_{1}(=a+h)$ versus $P$ assuming $Q=6$ and constant; at the right, similar plots considering $Q$ the variable and assuming $P=3$ and constant. As in Fig. 1 these graphs have been drawn using the program Microsoft Excel 4.0 for Macintosh. Values of the rate constants and $E t$ as in Fig. 1. As discussed in the text, $h, a$ and $b$ are parameters in an equation of type 3 that corresponds to a rectangular hyperbolic plot; $n_{1} / d_{1}$ is the value of the ordinate of the point where the horizontal asymptote of the rectangular hyperbola intercepts the vertical axis.

In absence of $\mathrm{P}, h=0$ and eqn. (13) turns into an equation of type 1 . In this case, the plot $v$ 
versus $A$ is an ascendant rectangular hyperbola that crosses the origin of the axes (Fig. 1; plot 2). Actually, this is just the Michaelis-Menten equation when the value of $b$ (the apparent $K_{\mathrm{m}}$ ) depends on $Q$ (eqn. 20).

At saturating concentrations of $\mathrm{P}$, the plot $v$ versus $A$ is an ascendant rectangular hyperbola $(a>0)$ with a negative value of the ordinate for $A=0(h<0)$, but the horizontal asymptote is the horizontal axis itself $\left(n_{1} / d_{1}=0\right.$; see Fig. 1; plot 3 ). In these conditions, $v$ is neg- ative even at high $A$ : $\mathrm{P}$ is a total inhibitor for the conversion of $\mathrm{A}$ into $\mathrm{P}+\mathrm{Q}$ and, as it does not compete with $\mathrm{A}$, saturating $P$ can not be overtaken by saturating $A$ [2].

(3) Influence of $\mathrm{Q}$ on the hyperbola parameters. If $Q$ is considered the variable in eqns. (14) and (15), it is easy to recognize them as equations of type 2. Note that for eqn. (14) $n_{0}=0$ and that eqn. (15) is, actually obtained by an addition of a constant value and an equation of type 2. These equations can be rearranged into equations of type 3 :

$h=\frac{-\left(\frac{k_{2} k_{4} P E t}{k_{2}+k_{3}+k_{4} P}\right) Q}{\left(\frac{\left(k_{2}+k_{3}\right) k_{5}+k_{2} k_{4} P}{\left(k_{2}+k_{3}\right) k_{6}+k_{4} k_{6} P}\right)+Q}$

$a=\frac{n_{1}}{d_{1}}-h=\frac{\frac{k_{3} k_{5} E t}{k_{4}}}{\frac{k_{3}+k_{5}}{k_{4}}+P}+\frac{\left(\frac{k_{2} k_{4} P E t}{k_{2}+k_{3}+k_{4} P}\right) Q}{\left(\frac{\left(k_{2}+k_{3}\right) k_{5}+k_{2} k_{4} P}{\left(k_{2}+k_{3}\right) k_{6}+k_{4} k_{6} P}\right)+Q}$

If $P$ is assumed constant, and a nonzero and finite quantity, from eqns. (22) and (23) it can be intuitively recognized that the plots $h$ versus $Q$ and a versus $Q$ are hyperbolas (see Fig. 2). As $Q$ increases from zero to saturation $(Q \rightarrow \infty)$ the value of $h$ decreases from zero to a finite negative quantity, the value of $a$ increases by a value that is equal to the decrease of $h$ so that $(a+h)$ remains constant; i.e. the position of the horizontal asymptote for the plot $v$ versus $A$ does not depend on $Q$ (see eqn. 17 and Fig. 1). Equation (16) shows that the plot $b$ versus $Q$ is a straight line $\left(d_{1}=0\right)$ the values of which for the ordinate at the origin and for the slope are always positive and depend on $P$ (see Fig. 2).

In absence of $\mathrm{Q}, h=0$ and eqn. (13) turns into an equation of type 1 and so the hyperbola crosses the origin of the axes; the value of $a$ is, in general, positive and so the hyperbola is as- cendant (Fig. 1; plot 2); again, it is just the Michaelis-Menten equation when the value of $b$ (the apparent $K_{\mathrm{m}}$; see eqn. 16 ) and the value of $a$ (the apparent $V_{\max }$; see eqn. 23) depend on $P$. In the particular case, in which $Q$ is absent and $P$ is saturating, the value of the parameter $a$ equals zero (see eqn. 23), and the plot $v$ versus $A$ is no more a hyperbola but a straight line coincident with the horizontal axis.

When $Q$ tends to infinity, $b$ tends to infinity (eqn. 16) and $a$ to a finite positive value that depends on $P$ (see eqn. 23); this means that at saturating $Q, v$ does not depend on $A$ unless $A$ is also saturating. Actually, $\mathrm{Q}$ is a competitive total inhibitor for the reaction $\mathrm{A} \rightarrow \mathrm{P}+\mathrm{Q}$ [2]: if $Q$ is supposed saturating, A can not bind to the enzyme; if $A$ is supposed saturating, $\mathrm{Q}$ can not bind to the enzyme [1]. So, if $Q$ is supposed saturating for all concentrations of $\mathrm{A}$, 
the plot $v$ versus $A$ is a straight line parallel to and located below the horizontal axis; the equation for this straight line is $v=h$ (Fig. 1; plot 4). If $Q$ is supposed saturating only at low concentrations of A (higher concentrations of A dislocate $\mathrm{Q}$ from the enzyme) the plot $v$ versus $A$ is an ascendant rectangular hyperbola intercepting the vertical axis below the origin of the axes; unless $P$ is saturating for all concentrations of $\mathrm{A}$, the hyperbola crosses the horizontal axis and tends to a positive value $\left(n_{1} / d_{1}\right)$ when $A$ tends to saturation (see Fig. 1 ; plot 1).

(4) Tridimensional representation. The classical Lineweaver-Burk (LB) plots can be made tridimensional by adding a third axis, representing the concentration of an inhibitor, perpendicular to both the LB axes and crossing them at point $(0,0)$ [3]. Similarly, the plot $v$ versus $A$ can be made tridimensional by adding a third axis representing $P$ or $Q$. The axes $A$ and $P$ (or $Q$ ) define a horizontal plane and the axes $v$ and $P$ (or $Q$ ) a vertical one. In this type of graph, a family of hyperbolas representing $v$ versus $A$ is obtained for different concentrations of the variable product. The points where the hyperbolas cross the vertical plane draw the plot $h$ versus $P$ (or $Q$ ); the points where the hyperbolas cross the horizontal plane draw the plot $\mathrm{A}_{\mathrm{eq}}$, versus $P$ (or $Q$ ).

\section{LINEAR TRANSFORMATIONS}

Several methods are commonly used to fit the experimentally obtained data to eqn. (1) by first transforming it into the equation of a straight line to which the data can be easily fitted by linear regression. Once equations of type 3 are obtained they can be easily turned into a linear form, the first step being to subtract $h$ from both terms of the equation:

$\mathrm{y}-h=\frac{a \mathrm{x}}{b+\mathrm{x}}$
Using the Lineweaver-Burk, Hanes, and Eadie-Hofstee methods [4-7], eqns. (25-27) can be deduced, respectively:

$\frac{1}{(\mathrm{y}-h)}=\frac{1}{a}+\frac{b}{a}(1 / \mathrm{x})$

$\frac{\mathrm{x}}{(\mathrm{y}-h)}=\frac{b}{a}+\frac{1}{a} \mathrm{x}$

$(\mathrm{y}-h)=a-b\left(\frac{\mathrm{y}-h}{\mathrm{x}}\right)$

So, although an equation of type $3[\mathrm{y}=f(\mathrm{x})]$ represents a hyperbolic function, straight lines are obtained when plotting $1 /(\mathrm{y}-h)$ versus $1 / \mathrm{x}, \mathrm{x} /(\mathrm{y}-h)$ versus $\mathrm{x}$, or $(\mathrm{y}-h)$ versus $(\mathrm{y}-h) / \mathrm{x}$. From these representations, as it is well known, graphical estimations of the parameters $a$ and $b$ can be obtained [4-7].

R.F. thanks the researchers of the Departments of Physiological Chemistry and Biochemistry (Universidade do Porto, Portugal) for facilitating his stay in Madrid. J.M.R. thanks Dr. J.C. Cameselle (Universidad de Extremadura, Spain) in whose laboratory part of this work was carried out.

\section{R E F E R E N C E S}

1. Fontes, R., Ribeiro, J.M. \& Sillero, A. (1999) Inhibition and activation of enzymes. The effect of a modifier on the reaction rate and on kinetic parameters. Acta Biochim. Polon. 47, 233-258.

2. Cleland, W.W. (1963) The kinetics of enzyme-catalyzed reactions with two or more substrates or products. I. Nomenclature and 
rate equations. Biochim. Biophys. Acta 67, 104-137.

3. Fontes, R., Ribeiro, J.M. \& Sillero, A. (1994) A tridimensional representation of enzyme inhibition useful for diagnostic purposes. $J$. Enzym. Inhib. 8, 73-85.

4. Lineweaver, H. \& Burk, D. (1934) The determination of enzyme dissociation constants. $J$. Am. Chem. Soc. 56, 658-666.

5. Hanes, C.S. (1932) CLXVII. Studies on plant amylases. I. The effect of starch concentration upon the velocity of hydrolysis by the amylase of germinated barley. Biochem. J. 26, 1406-1421.

6. Eadie, G.S. (1942) The inhibition of cholinesterase by physostigmine and prostigmine. J. Biol. Chem. 146, 85-93.

7. Hofstee, B.H.J. (1952) On the evaluation of the constants $V_{\mathrm{m}}$ and $K_{\mathrm{M}}$ in enzyme reactions. Science 116, 329-331. 